\title{
Sense p16 and antisense uPAR bicistronic construct inhibits angiogenesis and induces glioma cell death
}

\author{
NARASIMHARAO NALABOTHULA ${ }^{1}$, SAJANI S. LAKKA ${ }^{1}$, DZUNG H. DINH ${ }^{2}$, \\ MEENA GUJRATI $^{3}$, WILLIAM C. OLIVERO ${ }^{2}$ and JASTI S. RAO ${ }^{1,2}$ \\ ${ }^{1}$ Program of Cancer Biology, Department of Cancer Biology and Pharmacology, Departments of \\ ${ }^{2}$ Neurosurgery, and ${ }^{3}$ Pathology, University of Illinois College of Medicine at Peoria, Peoria, IL 61605, USA
}

Received August 25, 2006; Accepted November 2, 2006

\begin{abstract}
High-grade gliomas comprise the most malignant type of primary brain tumor and are relatively frequent in adults. Recent studies have indicated that the loss of p16, an inhibitor of CDK4, promotes the acquisition of malignant characteristics in gliomas. A correlation between overexpression of urokinase-type plasminogen activator receptor (uPAR) and glioblastoma invasion has also been established. Moreover, uPAR/integrin binding has been shown to initiate or potentiate integrin signaling through focal adhesion kinase and/or src kinases. Our previous studies demonstrated that downregulation of UPAR expression and restoration of p16 regress glioma growth in nude mice and downregulate $\alpha_{v} \beta_{3}$ integrin receptor expression. Here, we show the effect of a bicistronic construct on $\alpha_{v} \beta_{5}$ integrin receptor expression, angiogenesis and the biochemical pathway that causes glioma cell death. The U251 glioblastoma and a glioblastoma xenograft cell line transduced with a recombinant replication-defective adenovirus vector containing the cDNA of wild-type p16 and antisense RNA of UPAR significantly inhibited human mammary epithelial cell capillary formation and vascular endothelial growth factor (VEGF) expression. Inactivation of anti-apoptotic molecules such as Akt, PARP, activation of caspases and accumulation of heteroduplex chromosomal DNA in pre-G1 phase of the cell cycle was demonstrated by Western blotting, caspase activity assay and FACS analysis.
\end{abstract}

Correspondence to: Dr Jasti S. Rao, Department of Cancer Biology and Pharmacology, University of Illinois College of Medicine at Peoria, One Illini Drive, Peoria, IL 61605, USA

E-mail: jsrao@uic.edu.

Abbreviations: uPAR, urokinase plasminogen activator receptor; FAK, focal adhesion kinase; PI3K, phosphatidylinositol-3-kinase; VEGF, vascular endothelial growth factor; HMEC, human mammary epithelial cell; H\&E, hematoxylin and eosin; FACS, fluorescence activated cell sorter; RT-PCR, reverse transcriptase-polymerase chain reaction

Key words: p16 ${ }^{\mathrm{INK} 4 \mathrm{~A}}$, urokinase plasminogen activator receptor, angiogenesis, apoptosis, $\alpha_{v} \beta_{5}$ integrin receptor
Nuclear DNA fragmentation upon induction of apoptosis was scored using the TUNEL assay. Significant downregulation of $\alpha_{v} \beta_{5}$ integrin receptor expression was also confirmed by FACS analysis, immunoprecipitation and RT-PCR. Taken together, the results demonstrate that the sense p16 and anti-sense uPAR bicistronic construct significantly inhibits angiogenesis, induces apoptosis by deregulation of the PI3K-Akt pathway and downregulates $\alpha_{v} \beta_{5}$ integrin receptor expression.

\section{Introduction}

Gliomas account for $>50 \%$ of all brain tumors and are by far the most common primary brain tumors in adults (1). Genetic analysis suggests that there are at least two different types of glioblastoma: the first type occurs in elderly patients with de novo glioblastoma. The second type is rarer, occurs in younger patients and is usually evolved from low-grade (WHO grade II) or anaplastic astrocytomas (WHO grade III) $(2,3)$. Unfortunately, conventional therapies such as surgery, radiation, and chemotherapy have not shown much progress. Consequently, there is a necessity to develop new therapeutic strategies such as dual antagonists to eliminate gliomas completely.

Previous studies on glioblastomas have shown p16/ CDKN2A gene deletion in approximately $60 \%$ of glioblastomas (4) and $69-87 \%$ in glioma cell lines (5-8). The p $16^{\text {INK4a }}$ gene, which is located on chromosome 9 p 21 , encodes a protein of 156 amino acids comprising 4 ankyrin repeats. The main biological function of $\mathrm{p} 16^{\mathrm{INK} 4 \mathrm{a}}$ involves regulation of cell cycle progression at the G1/S boundary. In addition to the regulation of cell cycle progression, $\mathrm{p} 16^{\mathrm{INK} 4 \mathrm{a}}$ has been implicated in other fundamental cellular processes, such as angiogenesis (9), cell senescence (10), tumor invasion (11), cell spreading (12), and apoptosis $(13,14)$.

uPAR, a glycosylphosphatidylinositol-linked membrane protein, plays a pivotal role in glioma invasion by binding to uPA and focalizing proteolytic activity to the cell surface matrix (15). Our earlier findings suggest that the UPAR enhances glioma cell invasion, growth and angiogenesis (16). However, recent studies have revealed that the UPAR can form lateral associations with transmembrane integrins (17-23), and uPAR/integrin binding has been shown to 
initiate and/or potentiate integrin signaling through focal adhesion kinase and/or src kinases.

The formation of new blood vessels is essential for solid tumor growth and metastatic spread. A family of heterodimeric transmembrane adhesion receptors known as integrin receptors regulates these processes. The upregulation of integrins on glioma cells and the involvement of integrin receptors in the process of tumor astrocyte migration have been studied extensively. Immunohistochemical studies performed on frozen sections of glioblastoma periphery have revealed increased expression of VEGF and $\alpha_{\mathrm{V}} \beta_{5}$ integrin from the periphery to the tumor center. However, similar studies have demonstrated constant expression of FGF-2 and $\alpha_{V} \beta_{3}$ integrin (24). These findings suggest that FGF2 and VEGF may activate different angiogenic pathways that require $\alpha_{\mathrm{V}} \beta_{3}$ and $\alpha_{\mathrm{V}} \beta_{5}$ respectively.

Considering the roles of UPAR and p16 on glioma genesis, tumor invasion, angiogenesis and the impact of UPAR on integrin signaling, we hypothesized that restoration of p16 expression and downregulation of UPAR in glioblastoma cells through recombinant adenoviral constructs may provide better therapeutic strategies to eradicate malignant gliomas. Our previous studies have shown the anti-tumor and anti-invasion effects of the recombinant adenovirus carrying sense p16 and antisense uPAR (Ad-uPAR-p16) genes $(25,26)$. Here, we report the effects of the bicistronic construct on glioma angiogenesis, cell death, and $\alpha_{\mathrm{V}} \beta_{5}$ integrin receptor expression.

\section{Materials and methods}

Cell culture and adenovirus infection. U251 glioblastoma cancer cell line (U251) and glioblastoma xenograft cells (kindly provided by Dr David James, UCSF, San Francisco) were used in this study. U251 and glioblastoma xenograft cells were grown in RPMI-1640 and DMEM/F12 supplemented with $10 \%$ FBS. During the experimental period, cells were cultured in serum-free conditions on vitronectin-coated plates and chamber slides as described previously $(25,26)$. Briefly, after $24 \mathrm{~h}$ of culturing on vitronectin-coated 100 -mm tissue culture plates and chamber slides in serum-free conditions, cells were infected with recombinant adenoviral constructs such as Ad EV (empty vector), Ad uPAR (anti-sense uPAR), Ad p16 (sense p16), and Ad uPAR-p16 (anti-sense uPAR and sense p16) in serum-free medium for $72 \mathrm{~h}$.

Western blotting. $\mathrm{U} 251$ or glioblastoma xenograft cells $\left(1 \times 10^{6}\right)$, treated with $100 \mathrm{MOI}$ of one of the recombinant adenoviral constructs mentioned above, were sonicated in lysis buffer containing $50 \mathrm{mM}$ Tris- $\mathrm{HCl}, 150 \mathrm{mM} \mathrm{NaCl}, 1 \%$ sodium deoxycholate, $0.1 \%$ SDS, $20 \mathrm{mM}$ EDTA, $1 \mathrm{mM} \mathrm{NaF}, 1 \%$ Triton X-100 ( $\mathrm{pH} 7.4)$ as well as $1 \mathrm{mM}$ phenylmethylsulfonyl fluoride (PMSF), $10 \mu \mathrm{g} / \mathrm{ml}$ aprotinin and $1 \mathrm{mM}$ $\mathrm{Na}_{3}\left(\mathrm{VO}_{4}\right)$. Extraction buffer (0.1 M Tris, $\mathrm{pH} 7.5,1 \%$ Triton $\mathrm{X}-114,10 \mathrm{mM}$ EDTA, $1 \mathrm{mM}$ PMSF and $10 \mu \mathrm{g} / \mathrm{ml}$ aprotinin) was used to prepare lysates for UPAR immunoblotting. Equivalent amounts of total protein (30-50 $\mu \mathrm{g}$ per lane) were loaded onto 6-15\% acrylamide gels, resolved by electrophoresis and electrotransferred onto nitrocellulose membranes. Membranes were blocked in Blotto buffer (5\% skim milk in Tris-buffered saline-Tween) for $1 \mathrm{~h}$ and subsequently incubated with primary antibodies (mouse antiuPAR, anti-phospho-FAK, anti-FAK, anti-phospho-Akt, anti-PI3K, anti-caspase-9, anti-caspase-3, anti-VEGF and anti-GAPDH and rabbit anti-p16, anti-Akt, anti-Bcl-xL, antiBax, anti-pBAD, anti-BAD, anti-Cyt-C, anti-PARP; all at $1: 1000$ ) in Blotto for $14-16 \mathrm{~h}$ at $4^{\circ} \mathrm{C}$. Primary antibodies were detected with horseradish-peroxidase-conjugated goat IgG raised against the corresponding species in Blotto for $1 \mathrm{~h}$ at room temperature (goat anti-rabbit and goat anti-mouse; all at 1:2000). Peroxidase activity was detected using the ECL detection kit (GE Healthcare Bio-Sciences Corp., RPN-2106) and recorded on HyperFilm MP (GE Healthcare Bio-Sciences Corp., RPN-1678k). Protein content was normalized against GAPDH level, which was used as a loading control.

RNA isolation and semi-quantitative RT-PCR analysis. Total RNA from $1 \times 10^{6}$ cells treated with various viral constructs was isolated using the RNeasy Mini kit (Qiagen, Cat \#74134) and treated with RNAase-free DNAase to prevent genomic DNA contamination. Total RNA $(0.5-0.8 \mu \mathrm{g})$ was used for first-strand cDNA synthesis in a $50-\mu 1$ reaction volume. The reaction was performed with the aid of a Superscript One-Step RT-PCR System with Platinum Taq (Invitrogen Corporation, CA, Cat \#10928-042) kit per manufacturer's instructions. PCR products on $2 \%$ agarose gels were stained with ethidium bromide and visualized under UV trans-illumination. Primer pairs for cDNA amplification (in the 5'-3' direction) were as follows: 5'-CTGTTCTCGCTTCGGAGGAG-3' (forward) and 5'-CCCAAGACAGCAGAAAGTTCATGGTTT-3' (reverse) for human VEGF; 5'-GTTGGGAGATTAGACAGAGGA-3' (forward) and 5'-CAAAACAGCCAGTAGCAACAA-3' (reverse) for human $\alpha_{v}$ integrin subunit; 5'-ACTATCCATCC CTTGCCTTGCTTG-3' (forward) and 5'-GCAGTCCTTGGC GGTTTTGTAGAA-3' (reverse) for $\beta_{5}$ integrin subunit; and 5'-CCACCCATGGCAAATTCC-3' (forward) and 5'-CAGG AGGCATTGCTGATGAT-3' (reverse) for GAPDH. The expected fragment length of VEGF, $\alpha_{\mathrm{v}}$ and $\beta_{5}$ integrin subunits and GAPDH was 382, 288, 1127 and 298 bp, respectively.

FACS analysis. To analyze the impact of p16 expression and UPAR downregulation on glioma cell death, U251 or glioblastoma xenograft cells were treated with various recombinant adenoviral constructs as described previously. After $72 \mathrm{~h}$ of infection, cells were harvested, stained with propidium iodide and sorted for sub-diploid DNA (apoptotic cell) content on a Becton-Dickinson FACScalibur flow cytometer. To determine $\alpha_{\mathrm{V}} \beta_{5}$ integrin receptor expression, U251 or glioblastoma xenograft $\left(1 \times 10^{6}\right)$ cells treated with recombinant adenoviral constructs were harvested, fixed with $3 \%$ phosphate-buffered formalin at room temperature for $20 \mathrm{~min}$. These cells were resuspended in PBS buffer containing $1 \%$ BSA and incubated with anti- $\alpha_{\mathrm{v}} \beta_{5}$ antibody (R\&D Systems, MAB2528) or control (mouse IgG) antibody (Santa Cruz, sc-2025) at room temperature for $1.5 \mathrm{~h}$ with gentle agitation. After three washes in PBS buffer containing $1 \%$ BSA, cells were incubated with FITC-conjugated secondary antibody (Biomeda, SJ24065) at room temperature for $1 \mathrm{~h}$ in the dark with gentle rotation. Later, cells were washed twice, resuspended in PBS buffer and relative fluorescence was detected with an excitation wavelength of $488 \mathrm{~nm}$ and 
A

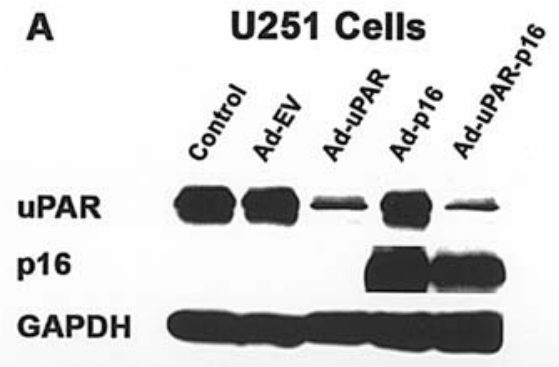

B Glioblastoma Xenograft Cells

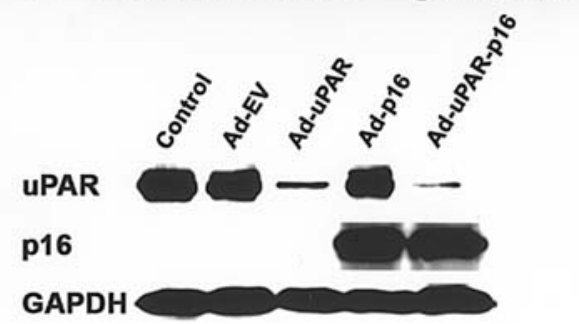

Figure 1. Western blot analysis of p16 expression and uPAR. U251 and glioblastoma xenograft cells were treated with $100 \mathrm{MOI}$ of each indicated recombinant virus and lysates were analyzed on Western blots for p16 expression and uPAR downregulation. (A) U251 cell lysates, treated with Ad-EV, Ad-uPAR, Ad-p16 and Ad-uPAR-p16, were probed with human anti-uPAR and anti p16 antibodies as described in Materials and methods. (B) Ad-EV, Ad-uPAR, Ad-p16 and Ad-uPAR-p16-treated glioblastoma xenograft cell lysates were probed with human anti-uPAR and anti-p16 antibodies as described above.

emission wavelengths of $525 \mathrm{~nm}$ for the Becton-Dickinson FACScalibur flow cytometer (Becton-Dickinson Immunocytochemistry Systems, CA).

Caspase 3 activity assay. Cell lysates were treated using a caspase 3 activity assay kit (EMD Biosciences, Catalog \#235418) according to the manufacturer's instructions. The assays are based on the spectrophotometric detection of chromophore p-nitroanilide (pNA) after cleavage from the labeled substrate DEVD-pNA. Comparison of the absorbance of pNA allows the determination of fold increase in caspase activity. Lysates $(25 \mu \mathrm{g})$ were added to the microtiter wells, and reaction was initiated by adding $200 \mu \mathrm{M}$ DEVD-pNA substrate. Protease activity was detected in a microplate reader at $405 \mathrm{~nm}$ after every $15 \mathrm{~min}$ of incubation at $30^{\circ} \mathrm{C}$. In parallel, 50 units of purified caspase 3 incubated with $200 \mu \mathrm{M}$ DEVDpNA substrate were used as a positive control and lysates incubated without DEVD-pNA substrate were used as a negative control. Assay buffer consisting of $200 \mu \mathrm{M}$ DEVDpNA substrate was considered as a blank. Each reaction was performed in quadruplicate and linear regression analysis was used to obtain the slope. Average $\mathrm{A}_{405}$ of $50 \mu \mathrm{M}$ p-nitroaniline (pNA) in assay buffer was subtracted with average $\mathrm{A}_{405}$ of the blank and divided by the concentration of pNA $(50 \mu \mathrm{M})$ and the product was considered as a conversion factor. Graphs were constructed with caspase activities calculated as follows: activity $(\mathrm{pmol} / \mathrm{min})=$ slope of sample reaction $\mathrm{x}$ conversion factor $\mathrm{x}$ assay volume (100 $\mu \mathrm{l})$.

TUNEL assay. To evaluate the apoptotic response of the uPAR-p16 bicistronic construct, we applied the terminal deoxynucleotidyl transferase (TdT)-mediated biotin-dUTP nickend labeling (TUNEL) technique using the commercially available TUNEL apoptosis detection kit (Upstate USA, Catalog \#17-141). Briefly, 1.0x106 cells were seeded on vitronectin-coated chamber slides and infected with Ad-EV, Ad-uPAR, Ad-p16 and Ad-uPAR-p16 constructs as mentioned previously. After $72 \mathrm{~h}$, cells were washed with PBS and fixed with $3 \%$ phosphate-buffered formalin. These cells were washed in PBS and incubated with $0.05 \%$ Tween-20, 0.2\% BSA in PBS for $15 \mathrm{~min}$ at room temperature. After washing in PBS, cells were incubated with TdT end-labeling cocktail for $1 \mathrm{~h}$ and then the reaction was stopped with TB buffer. Incorporated biotin-dUTP was detected under a fluorescence microscope after probing with FITC-conjugated avidin antibody.
In vitro angiogenesis assay. U251 or glioblastoma xenograft cells $\left(2 \times 10^{4}\right)$ were seeded per well of eight-well chamber slides and infected with the indicated MOI of Ad-EV, Ad-uPAR, Ad-p16 and Ad-uPAR-p16. Infected cells were incubated for $24 \mathrm{~h}$, medium was removed, and $4 \times 10^{4}$ human dermal endothelial cells (HMEC) suspended in endothelial cell growth medium were seeded. After $72 \mathrm{~h}$ of incubation, cells were fixed in $3 \%$ phosphate-buffered formalin, blocked in $3 \% \mathrm{BSA}$ in PBS and stained for factor VIII antibody (Dako Corporation, Carpinteria, CA). Factor VIII-stained cells were washed with PBS and incubated with FITC-conjugated secondary antibody in the dark for $1 \mathrm{~h}$. These cells were then washed and endothelial cell tube formation was examined under a laser scanning confocal microscope. For H\&E staining, conditioned medium was collected from cells infected with the above recombinant viral constructs for $48 \mathrm{~h}$. This medium was added to the HMEC $\left(4 \times 10^{4}\right)$ cell monolayer and cultured for $72 \mathrm{~h}$. Thereafter, cells were stained with hematoxylin and eosin and endothelial cell network formation was monitored under a laser scanning confocal microscope.

Immunoprecipitation. Surface-expressed proteins on U251 and glioblastoma xenograft cells were labeled as described by Milner et al $(27,28)$. Briefly, $1 \times 10^{6}$ cells (infected with 100 MOI of Ad-EV, Ad-uPAR, Ad-uPAR-p16 and Ad-p16 constructs for $72 \mathrm{~h}$ ) were washed and labeled with $0.1 \mathrm{mg} / \mathrm{ml}$ NHS-LC-Biotin (Pierce) in PBS at $37^{\circ} \mathrm{C}$ in a $5 \% \mathrm{CO}_{2}$ atmosphere for $1 \mathrm{~h}$. Labeled cells were washed and lysed in extraction buffer for $1 \mathrm{~h}$ on ice, followed by trituration and centrifugation at $14,000 \mathrm{rpm}$ at $4^{\circ} \mathrm{C}$. Supernatants were pre-cleared by incubation with Protein A/G PLUS-Agarose (Santa Cruz Biotechnology, \#sc-2003) followed by mouse IgG. Immunoprecipitations were carried out overnight at $4^{\circ} \mathrm{C}$ on a rotating platform using $1 \mu 1$ anti-integrin $\alpha_{\mathrm{V}} \beta_{5}$ monoclonal antibody/100 $\mu 1$ cell lysate (R\&D Systems, MAB2528). The immune complexes were allowed to bind to the protein A/G PLUS-Agarose (20 $\mu \mathrm{l}$ slurry) for $1 \mathrm{~h}$ at $4^{\circ} \mathrm{C}$. Immunoprecipitates were collected by centrifugation at 2,500 rpm for $5 \mathrm{~min}$ at $4^{\circ} \mathrm{C}$. Pellets were washed in PBS four times, each time repeating the centrifugation step described above. After the final wash, the pellet was resuspended in $40 \mu 1$ of $1 \mathrm{X}$ sample buffer, boiled for $3 \mathrm{~min}$ and $20 \mu \mathrm{l}$ of the sample was analyzed by SDS-PAGE, electroblotting and biotin-labeled proteins were detected with streptavidin-HRP on Hyper Film MP using an ECL detection kit (Amersham). 


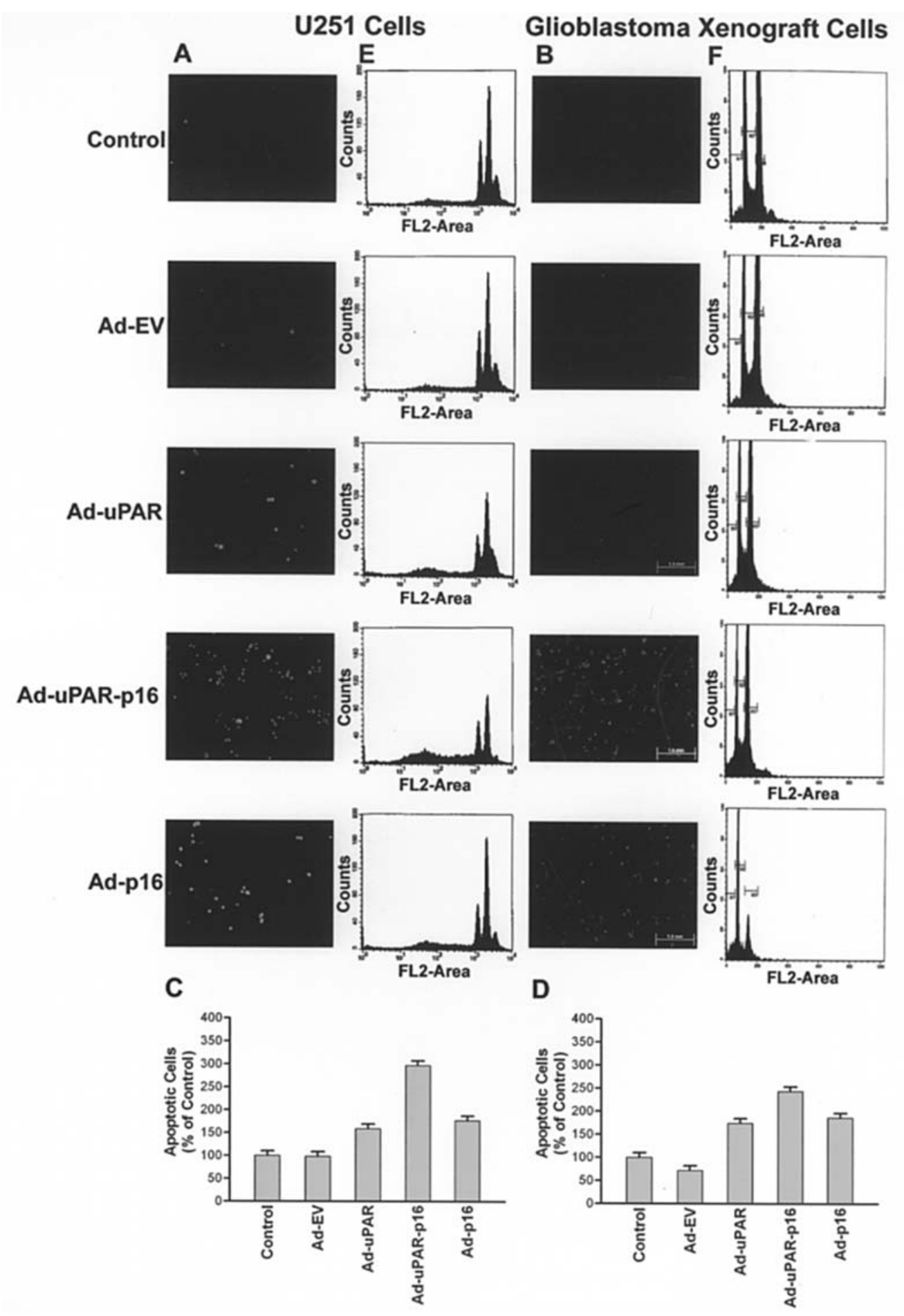

Figure 2. Ad-uPAR-p16 bicistronic construct induces U251 and glioblastoma xenograft cell death. In situ DNA end-labeling. U251 and glioblastoma xenograft cells were infected with the indicated viruses. Later, fixed cells were washed and incubated with TdT end-labeling cocktail for $1 \mathrm{~h}$ and then the reaction was stopped with TB buffer. Fluorescence-stained DNA strand breaks were detected using fluorescence microscopy after probing with FITCconjugated avidin antibody. (A and B) Fluorescence images of untreated and treated U251 and glioblastoma xenograft cells subjected to TUNEL assay, respectively. FACS analysis: U251 and glioblastoma xenograft cells were treated with the indicated viruses. These cells, harvested by trypsinization, were then stained with PI and apoptosis was determined by FACS analysis. (C and D) Histograms generated by plotting the sub-G1 population of untreated cells versus the sub-G1 population of treated cells. Data are expressed as the means \pm SD of three independent experiments. (E and F) Representative flow cytometry histograms.

\section{Results}

uPAR-p16 bicistronic construct restores p16 expression and specifically knocks down UPAR expression. To express p16 exogenously and inhibit uPAR expression, we used recombinant adenovirus carrying p16 cDNA and UPAR anti- sense RNA made by Adachi et al (26). Transfection of cells with Ad-p16, Ad-uPAR and Ad-uPAR-p16 resulted in the overexpression of $\mathrm{p} 16$, a reproducible decrease in $\mathrm{UPAR}$ expression in U251 and glioblastoma xenograft cell lines as demonstrated by Western blotting (Fig. 1). Empty vector (EV) carrying neither p16 cDNA nor uPAR anti-sense RNA 

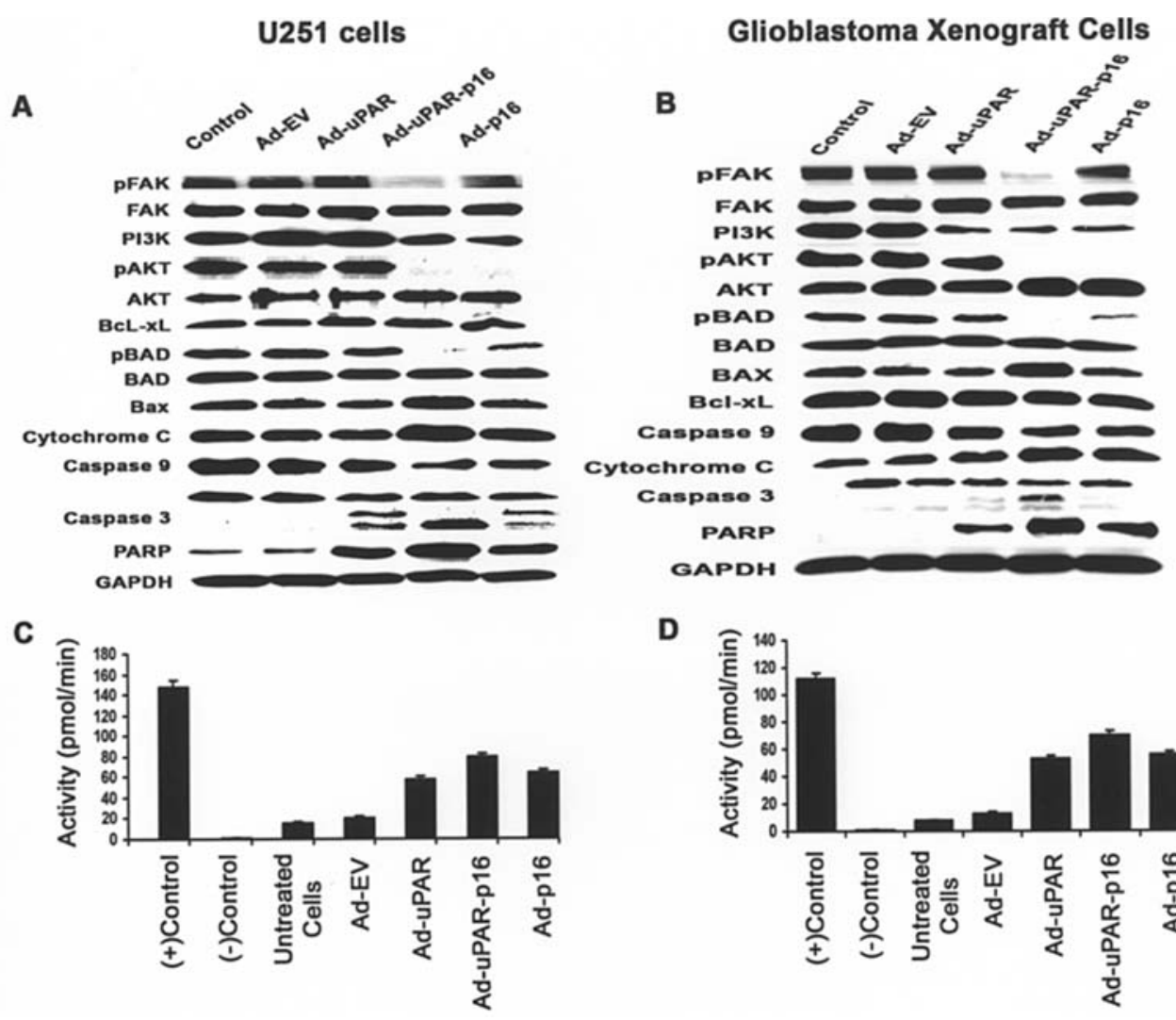

D

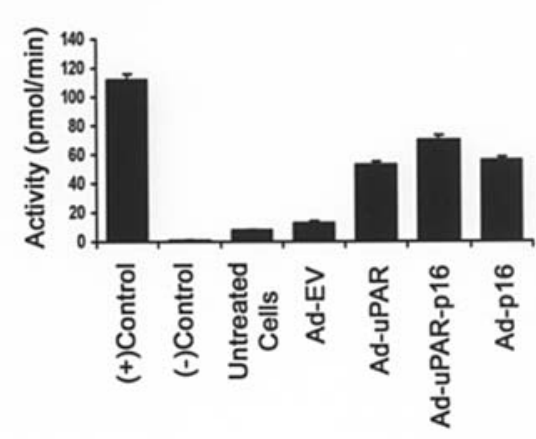

Figure 3. Effect of uPAR-p16 bicistronic construct on PI3K-Akt signal transduction pathway. Cell lysates of serum-starved U251 and glioblastoma xenograft cells infected with Ad-EV, Ad-uPAR, Ad-uPAR-p16 and Ad-p16 were prepared. Active and inactive forms of molecules involved in the PI3K/Akt cell survival pathway molecules were detected on Western blots as described in Materials and methods. Western blot analyses of molecules involved in the PI3K-Akt pathway in (A) U251 cells and (B) glioblastoma xenograft cells. Quantitative estimation of caspase 3 activity. Cell lysates prepared from various recombinant virus-treated cells were quantitated for active caspase 3 levels using spectrophotometric detection of chromophore p-nitroanilide (pNA) at 405 nm as described in Materials and methods. Caspase 3 activity (pmol/min) was plotted against cell lysates treated with indicated virus. Data represent \pm SD from three independent experiments performed in quadruplicate. Histograms represent quantitative estimation of active caspase 3 levels in treated and untreated (C) U251 cells and (D) glioblastoma xenograft cells. Reaction consisting of $200 \mu \mathrm{M}$ DEVD-pNA substrate and 50 units of purified caspase 3 was used as a positive control and lysates containing no DEVD-pNA were used as a negative control.

failed to show any impact on either p16 expression or uPAR downregulation. The effect of recombinant adenoviral constructs used was highly specific in that none of them showed affected unrelated protein GAPDH.

Apoptosis in bicistronic construct-treated glioma cells. Chromosomal DNA breakage and accumulation of damaged DNA in the pre-G1 phase of the cell cycle is a hallmark of apoptotic cells. Thus, we quantitated DNA damage using Biotin-dUTP labeling (TUNEL assay) and the population of cells containing semi-diploid DNA (FACS analysis). As shown in Fig. 2, a remarkably high number of cells treated with the Ad-uPAR-p16 bicistronic construct were TUNELpositive. Some of the cells treated with Ad-uPAR and Adp16 were TUNEL-positive, but the number was considerably less than that of the bicistronic construct-treated cells. In contrast, untreated cells and cells treated with Ad-EV (empty vector) were completely TUNEL-negative. Propidium iodidestained cells analyzed on FACS (Fig. 2C and D) demonstrated that in the presence of a bicistronic construct, the number of apoptotic cells was almost three times more than untreated or cells treated with the empty vector (Ad-EV). The bicistronic construct has demonstrated more apoptotic potential than either of the Ad-uPAR or Ad-p16 constructs. These results suggest that the glioma cells are prone to apoptosis in the presence of p16 and in the absence of UPAR.

UPAR-p16 bicistronic construct induces glioma cell death by inhibiting the PI3K-Akt pathway. Because glioma tumor cells survive by activating the PI3K-Akt cell survival pathway constitutively, we chose to analyze this pathway in recombinant adenovirus-treated U251 and glioblastoma xenograft cells following culture on vitronectin-coated plates. Inactivation of PI3K-Akt pathway and induction of caspasemediated intrinsic cell death were determined by comparing the amount of phospho proteins with the total amount of protein present and cleaved fragments with the amount of full-length protein. As shown in Fig. 3A and B, the Ad-uPARp16 bicistronic construct significantly dephosphorylated FAK, Akt, and BAD proteins. In contrast, the extent of dephosphorylation of FAK, Akt, and BAD proteins in Ad-uPAR and Ad-p16-treated cells was comparatively less than in bicistronic construct-treated cells. Furthermore, untreated cells and cells treated with Ad-EV (empty vector) showed no effect on the phosphorylation status of these proteins. Bicistronic construct-treated cells showed a slight increase in Bax protein expression, whereas no detectable change in $\mathrm{BcL}-\mathrm{xL}$ protein expression was observed in cells treated with 


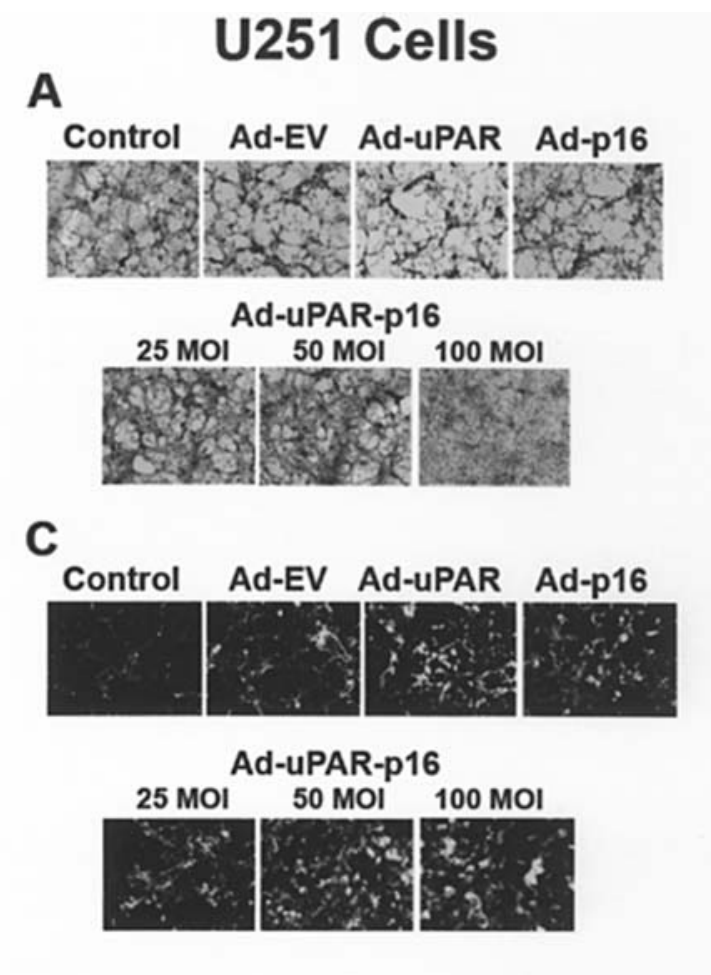

\section{Glioblastoma Xenograft Cells} B

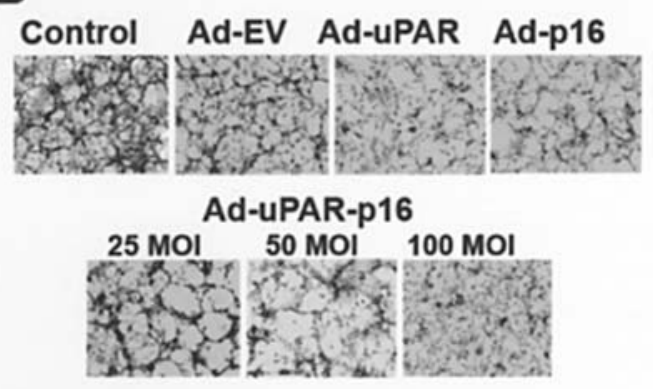

D
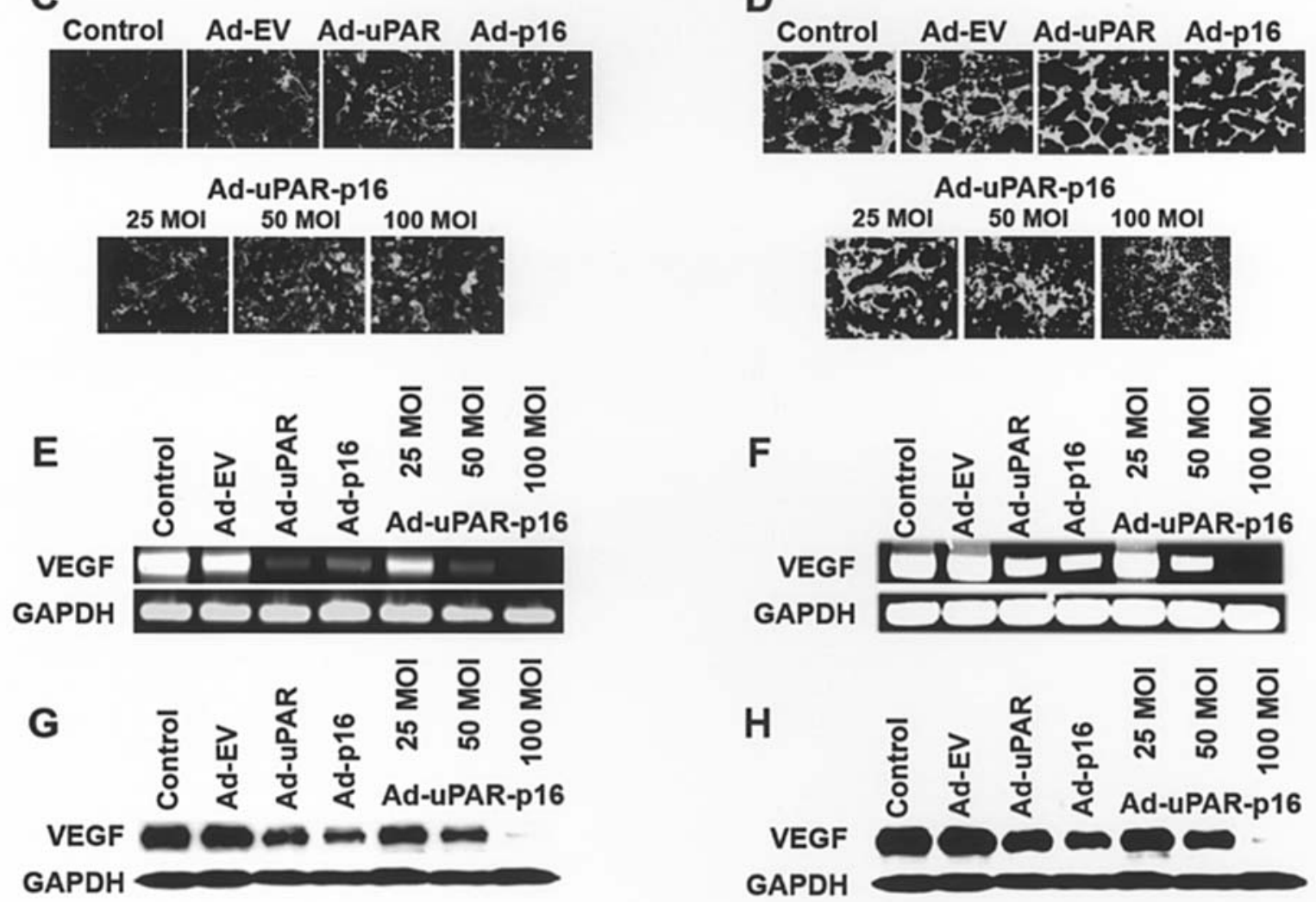

Figure 4. In vitro angiogenesis. H\&E staining: endothelial cells were grown in conditioned medium, collected from U251 and glioblastoma xenograft cells infected with indicated adenoviral constructs as described in Materials and methods, for $72 \mathrm{~h}$. Then, the cells were stained with hematoxylin and eosin and capillary network formation was observed under a laser scanning confocal microscope. (A) Endothelial cell network formation in the presence of conditioned medium collected from indicated recombinant virus-treated U251 cells. (B) Endothelial cell network formation in the presence of conditioned medium collected from indicated recombinant virus-treated glioblastoma xenograft cells. Factor VIII staining: U251 and glioblastoma xenograft cells, seeded in eightwell chamber slides, were incubated overnight and infected with the indicated MOI of Ad-EV, Ad-uPAR, Ad-uPAR-p16 or Ad-p16 virus. After a 24-h infection period, the cells were co-cultured with HMEC cells in endothelial cell growth medium for $72 \mathrm{~h}$. Then, the HMEC cells were stained for endothelial cell marker (factor VIII) and network formation was examined under a confocal scanning laser microscope. (C) U251 cells treated with indicated recombinant virus were co-cultured with HMEC cells and stained for factor VIII. (D) Glioblastoma xenograft cells treated with indicated recombinant virus were cocultured with HMEC cells and stained for factor VIII. RT-PCR analysis of VEGF mRNA expression in the presence of indicated recombinant virus in U251 (E) and glioblastoma xenograft cells (F), respectively. Western blot analyses of VEGF protein expression in U251 (G) and glioblastoma xenograft cells (H) treated with the indicated recombinant adenovirus, respectively.

any of the above constructs. In subsequent experiments, significant increase in cytoplasmic cytochrome $\mathrm{C}$, processing of caspase 9 and 3 into active forms, and cleavage of PARP were observed in Ad-uPAR-p16-treated cells as compared to untreated cells. However, cells treated with either Ad-p16 or Ad-uPAR showed a similar effect but the effect was considerably less than in cells treated with the Ad-uPAR-p16 bicistronic construct. The Ad-EV (empty vector) had no effect on the activity of any of the above proteins. This implies that glioblastoma cells under study (U251 and glioblastoma xenograft cells) undergo apoptosis upon restoration of p16 and downregulation of UPAR by inactivating the PI3K-Akt cell survival pathway and inducing the mitochondrial-dependent intrinsic apoptotic pathway.

Restoration of p16 and downregulation of uPAR inhibits angiogenesis. Neovascularization is needed for oxygenation and growth of solid tumors. Moreover, gliomas have been 

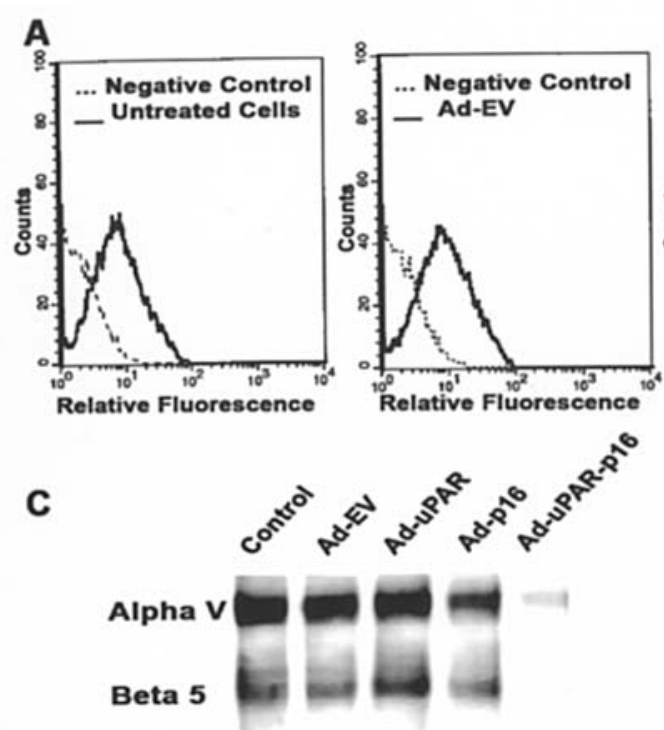

\section{U251 Cells}
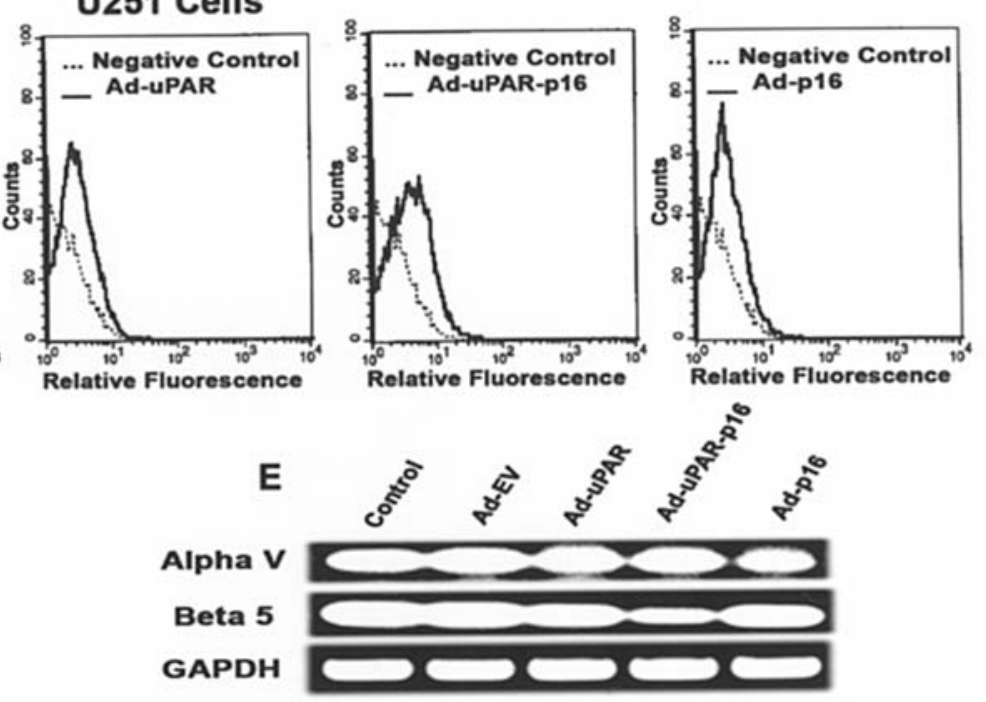

B

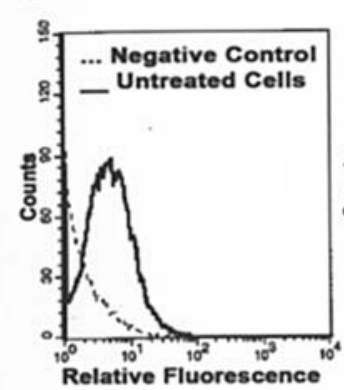

D

\section{Glioblastoma Xenograft Cells}
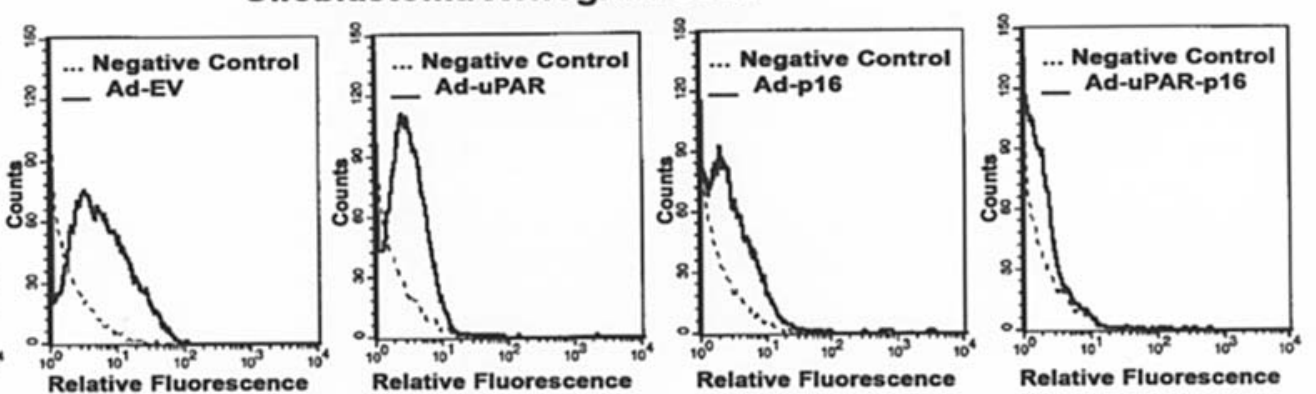
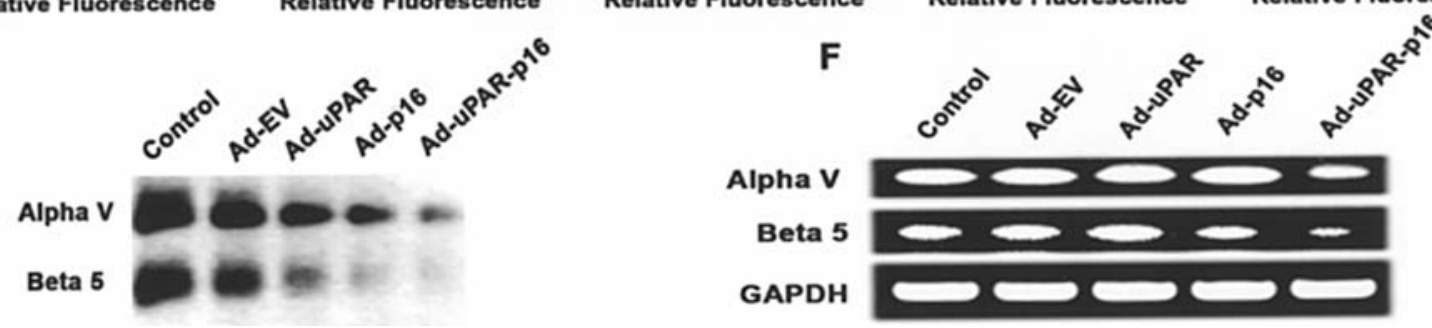

Figure 5. uPAR-p16 bicistronic construct downregulates $\alpha_{v} \beta_{5}$ integrin receptor expression. (A) U251 and glioblastoma xenograft cells were infected with the indicated virus and the surface-localized $\alpha_{v} \beta_{5}$ integrin receptor was probed with anti- $\alpha_{v} \beta_{5}$ integrin monoclonal antibody incubation. After incubation with FITC-conjugated goat anti-mouse secondary antibody, cell surface expression levels of $\alpha_{v} \beta_{5}$ integrin receptor were analyzed by measuring relative fluorescence intensity on a Becton-Dickinson FACScalibur flow cytometer. (A and B) Representative flow cytometric histograms for $\alpha_{v} \beta_{5}$ integrin receptor in U251 and glioblastoma xenograft cells, respectively. Immunoprecipitation analysis of $\alpha_{v} \beta_{5}$ integrin expression: cell lysates, prepared from the indicated recombinant adenovirus-infected cells, were immunoprecipitated with anti-integrin $\alpha_{v} \beta_{5}$ antibody. Biotin-labeled immune complexes purified from protein A/G PLUS-Agarose were detected with streptavidin-HRP on Hyper Film MP with the aid of an ECL detection kit. $\alpha_{v} \beta_{5}$ integrin receptor protein expression in U251 (C) and glioblastoma xenograft cella (D), respectively. RT-PCR analysis of $\alpha_{v} \beta_{5}$ mRNA expression. Total RNA of cells treated with various recombinant viral constructs were subjected to RT-PCR using a Superscript One-step RT-PCR System with Platinum Taq kit. See Materials and methods for details. (E) RT-PCR analysis of $\alpha_{\mathrm{v}} \beta_{5}$ integrin mRNA expression in U251 cells. (F) RT-PCR analysis of $\alpha_{\mathrm{v}} \beta_{5}$ integrin mRNA expression in glioblastoma xenograft cells. Housekeeping gene (GAPDH) mRNA expression levels were used as controls.

reported to be angiogenic. Thus, we have explored the impact of the bicistronic construct on glioma angiogenesis. Endothelial cells, grown in the presence of U251 or glioblastoma xenograft cells, exhibited extensive capillary-like structure formation (Fig. 4A-D). In comparison, capillary-like structure formation by endothelial cells was progressively decreased in bicistronic construct-treated U251 or glioblastoma xenograft cells in a dose-dependent manner. Ad-uPAR-p16 (100 MOI) inhibited capillary formation by $100 \%$; 100 MOI of Ad-uPAR and Ad-p16 repressed capillary formation by $\sim 65 \%$ and $75 \%$ of control subsequently. However, Ad-EV (empty vector) had no effect on endothelial cell capillary formation.
VEGF is a widely studied growth factor for neovascularization. As such, we tested VEGF expression in the presence of the Ad constructs. RT-PCR analysis revealed downregulation of VEGF mRNA expression in both cell lines treated with the bicistronic construct (Fig. 4E and F). Cells treated with $100 \mathrm{MOI}$ of the bicistronic construct expressed significantly lower levels of VEGF mRNA than cells treated with $100 \mathrm{MOI}$ of either anti-sense uPAR or sense p16 constructs. Besides, Western blots indicated decreased amounts of VEGF protein (Fig. 4G and $\mathrm{H}$ ) expression in a dose-dependent manner in both U251 cells and glioma xenograft cells infected with the bicistronic construct. Thus, the effect of the bicistronic 


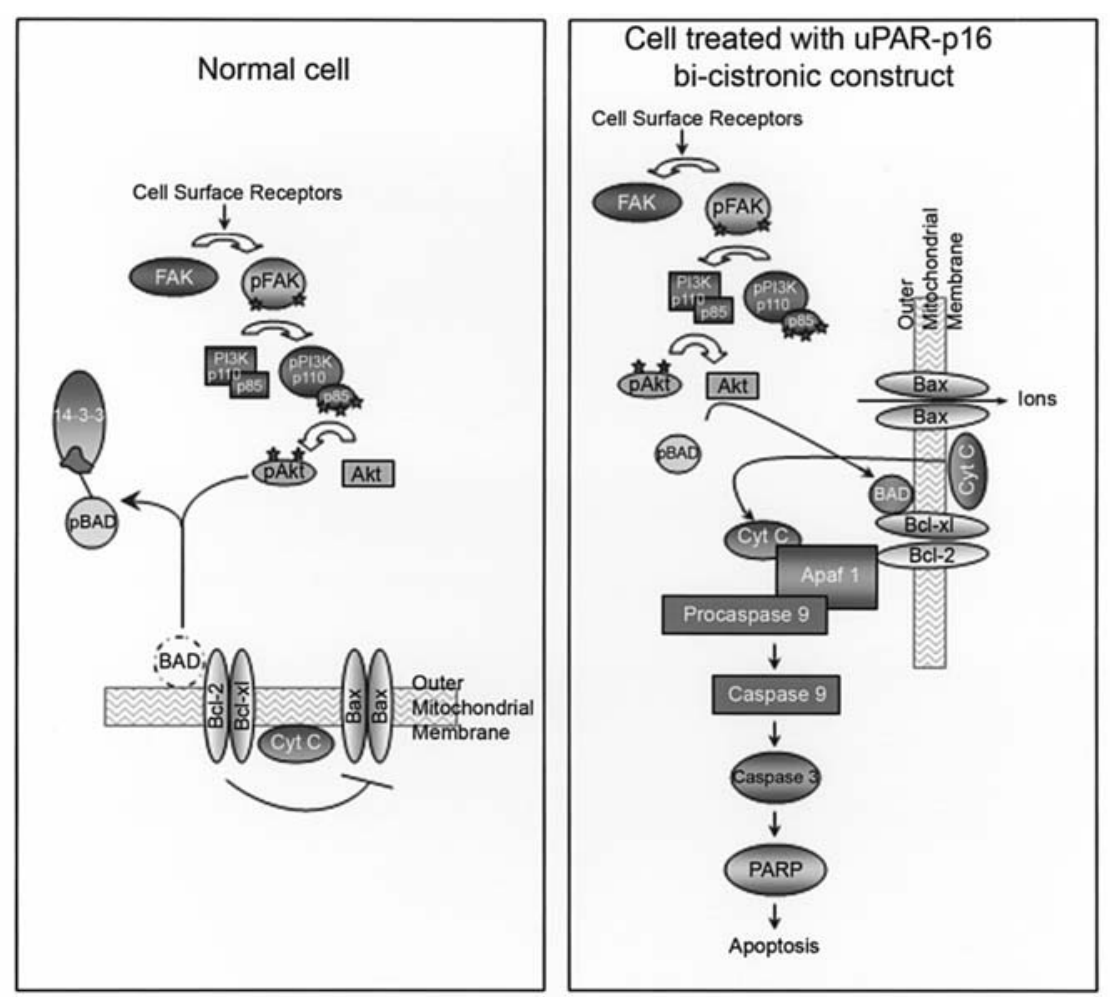

Figure 6. Schematic representation of Ad-uPAR-p16 mediated apoptotic pathway in glioma cells. Tumor cell survival mechanism by Akt-mediated BAD phosphorylation followed by 14-3-3 chaperon-mediated pBAD degradation (left). Tumor cell death mechanism in the presence of sense-p16 and antisenseuPAR bicistronic construct via inhibition of pAkt-mediated BAD phosphorylation followed by activation of caspase-mediated cell death (right).

construct on angiogenesis was more prominent than that of either Ad-p16 or Ad-uPAR. These results further support the anti-angiogenic effect of Ad-uPAR-p16.

uPAR-p16 bicistronic construct downregulates $\alpha_{V} \beta_{5}$ integrin expression. In the present study, the expression profile of $\alpha_{\mathrm{V}} \beta_{5}$ integrin was characterized by FACS, RT-PCR and immunoprecipitation analyses. Untreated or Ad-EV-infected cells (U251 or glioblastoma xenograft cells) emitted significantly higher amounts of fluorescence than background fluorescence. On the other hand, cells treated with the bicistronic construct emitted an almost equal amount to the background fluorescence. These observations demonstrated significantly less $\alpha_{\mathrm{V}} \beta_{5}$-positive cells in Ad-uPAR-, Ad-p16- and Ad-uPAR-p16treated cells as compared to uninfected or Ad-EV-infected controls (Fig. 5A and B). Both cell lines (U251 and glioblastoma xenograft cells) showed considerable downregulation of $\alpha_{\mathrm{V}} \beta_{5}$ protein expression (Fig. 5C and D) as well as mRNA expression (Fig. 5E and $\mathrm{F}$ ) in the presence of the bicistronic construct. The $\alpha_{\mathrm{V}} \beta_{5}$ integrin protein and mRNA expression profiles further support the proposition that the bicistronic construct downregulated $\alpha_{V} \beta_{5}$ integrin receptor expression in glioma cells.

\section{Discussion}

Glioblastomas are highly malignant tumors of the central nervous system that are resistant to radiation and chemotherapy. Initial efforts, focused on the use of single agents, directed at specific molecular targets have been disappointing. Moreover, it is clear that the complexity and cross-talk between signal transduction pathways limits the potential efficacy of targeting a single receptor or molecule. Therefore, the development of a combination of molecularly based therapies with multiple inhibitors or a combination of these pathway-specific treatments for gliomas holds the promise for an effective means of combating the disease. Two fields that are particularly important in this regard are angiogenesis, where the hope is that interfering in the tumor's blood supply will not only remove existing lesions but also prevent their recurrence, and apoptosis, which would produce particularly potent therapies if one could selectively turn on the machinery of cellular self-destruction in the cancer. p16 has been known to regulate the G1-S phase transition of the cell cycle, angiogenesis and UPAR is needed for glioma tumor metastasis. Hence, we designed an adenoviral bicistronic construct (Ad-uPAR-p16), which expresses p16 and downregulates UPAR, to induce glioma cell death and inhibit angiogenesis. We have already shown that the Ad-uPAR-p16 bicistronic construct regresses glioma tumors and inhibits tumor invasion $(25,26)$. In the present study, we report the effect of the bicistronic construct on glioma angiogenesis, integrin receptor expression and the molecular mechanism that turns off the glioma self-defense mechanism.

A number of recent studies demonstrated that PI3K-Akt pathway prevents glioma cell apoptosis. As such, we presumed that the Ad-uPAR-p16 bicistronic construct may cause glioma cell death through inhibition of the PI3K-Akt cell survival pathway. In the present study, we observed significant 
dephosphorylation of FAK, Akt, BAD, and activation of the caspase pathway (i.e., release of cytochrome $\mathrm{C}$, activation of caspases 9 and 3, and inactivation of PARP) in Ad-uPAR-p16treated cells. We also observed increased accumulation of sub-diploid DNA in pre-G1 phase of the cell, elevated active caspase 3 levels and TUNEL-positive cells after treatment with Ad-uPAR-p16. A central control point for the induction of apoptosis is the permeabilization of the mitochondrial outer membrane potential (MOMP), leading to the release of cytochrome $\mathrm{C}$ and other proteins residing in the mitochondrial intermembrane space. Within a short time, MOMP is followed by apoptosome formation and caspase activation resulting in apoptosis (29). BAD is one member of a group of 'BH3 domain only' proteins that appears to transduce death signals via heterodimerization to the Bcl-XL (anti-apoptotic) family of death regulators (30-33). However, Akt (a cell survival factor) has been reported to prevent permeabilization of MOMP by phosphorylation and inhibition of the proapoptotic effect of BAD (34-36). Recent studies have shown FAK/PI3K complex formation and suggested that FAK is the upstream signal protein of the PI3K-Akt pathway in glioblastoma cells (37-39). Based on the above available information and our present observations, we conclude that the Ad-uPAR-p16 bicistronic induces glioblastoma cell apoptosis through dephosphorylation of FAK, Akt, BAD and induction of the mitochondrial-dependent intrinsic caspase pathway (Fig. 6).

A variety of factors including vascular endothelial growth factor (VEGF), platelet derived growth factor (PDGF), fibroblast growth factor (FGF), and angiopoietins have been suggested to play a role in angiogenesis in solid tumors. Among all of these factors, VEGF has been identified as a potential tumor angiogenesis factor in human gliomas (40). Since angiogenesis is needed for the survival of solid tumors, targeting angiogenesis would certainly provide a better answer for glioma therapy. Here, we studied the effect of Ad-uPAR-p16 on glioma angiogenesis in addition to its ability to induce glioma cell death. To ascertain the effect of Ad-uPAR-p16 on angiogenesis, we performed a tube assay and tested expression levels of VEGF, which induces neovascularization, at the mRNA and protein levels. Earlier studies have shown partial inhibition of glioma tumor angiogenesis upon restoration of p16 (9). In the present study, endothelial cells grown in the presence of conditioned medium collected from Ad-uPAR-p16-treated glioblastoma cells did not form capillary-like structures. Moreover, we observed complete downregulation of VEGF mRNA as well as VEGF protein in the presence of Ad-uPAR-p16. Based on these results, we report that Ad-uPAR-p16 completely inhibits glioma tumor angiogenesis.

Several studies have investigated the role of integrins in glioma tumor metastasis, angiogenesis and signal transduction. A study by Bello et al (24) showed a correlation between $\alpha_{V} \beta_{5}$ integrin and VEGF, a pro-angiogenic molecule, expression. In the present study, using FACS, RT-PCR and immunoprecipitation analyses, we also observed the downregulation of $\alpha_{V} \beta_{5}$ integrin receptor expression in the presence of Ad-uPAR-p16. The inhibition of endothelial capillary-like structure formation, the downregulation of VEGF and $\alpha_{\mathrm{V}} \beta_{5}$ integrin receptor expression upon restoration $\mathrm{p} 16$, and the antisense-mediated downregulation of uPAR suggest that the Ad-uPAR-p16 bicistronic construct inhibits glioma tumor angiogenesis through downregulation of $\alpha_{V} \beta_{5}$ integrin receptor expression.

Based on all of these results, we conclude that Ad-uPARp16-mediated downregulation of UPAR and restoration of p16 expression in gliomas induces glioma cell apoptosis through inhibition of the PI3K-Akt self defense mechanism and induction of caspase-mediated mitochondrial intrinsic apoptotic pathway. Moreover, these results also suggest that Ad-uPAR-p16 inhibits angiogenesis and downregulates $\alpha_{\mathrm{V}} \beta_{5}$ integrin receptor expression in glioblastoma cells.

\section{Acknowledgements}

We thank Shellee Abraham for assistance in manuscript preparation and Diana Meister and Sushma Jasti for manuscript review. This research was supported by National Cancer Institute grants CA 75557, CA 92393, CA 95058, CA 116708 and N.I.N.D.S. NS47699 and Caterpillar, Inc., OSF Saint Francis, Inc., Peoria, IL (J.S.R.).

\section{References}

1. Kleihues P and Cavenee WK: In: Pathology and Genetics of Tumours of the Central Nervous System. P Kleihues and WK Cavenee (eds.). World Heath Organization Classification of Tumours. IARC, Lyon, 2000.

2. Tohma Y, Gratas C, Biernat W, Peraud A, Fukuda M, Yonekawa Y, Kleihues P and Ohgaki H: PTEN (MMAC1) mutations are frequent in primary glioblastomas (de novo) but not in secondary glioblastomas. J Neuropathol Exp Neurol 57: 684-689, 1998.

3. Von Deimling A, von Ammon K, Schoenfeld D, Wiestler OD, Seizinger BR and Louis DN: Subsets of glioblastoma multiforme defined by molecular genetic analysis. Brain Pathol 3: 19-26, 1993 .

4. Ohgaki H, Schauble B, zur HA, von AK and Kleihues P: Genetic alterations associated with the evolution and progression of astrocytic brain tumours. Virchows Arch 427: 113-118, 1995.

5. Arap W, Nishikawa R, Furnari FB, Cavenee WKS and Huang HJ: Replacement of the p16/CDKN2 gene suppresses human glioma cell growth. Cancer Res 55: 1351-1354, 1995.

6. Gomi A, Sakai R, Ogawa S, Shinoda S, Hirai H and Masuzawa T: human gliomas. Jpn J Cancer Res 86: 342-346, 1995.

7. He J, Allen JR, Collins VP, Lalunis-Turner MJ, Godbout R, Day RS III and James CD: CDK4 amplification is an alternative mechanism to p16 gene homozygous deletion in glioma cell lines. Cancer Res 54: 5804-5807, 1994.

8. Kamb A, Shattuck-Eidens D, Eeles R, Liu Q, Gruis NA, Ding W, Hussey C, Tran T, Miki Y, Weaver-Feldhaus J and : Analysis of the p16 gene (CDKN2) as a candidate for the chromosome 9p melanoma susceptibility locus. Nat Genet 8: 23-26, 1994.

9. Harada H, Nakagawa K, Iwata S, Saito M, Kumon Y, Sakaki S, Sato K and Hamada K: Restoration of wild-type p16 downregulates vascular endothelial growth factor expression and inhibits angiogenesis in human gliomas. Cancer Res 59: 3783-3789, 1999.

10. Vojta PJ and Barrett JC: Genetic analysis of cellular senescence. Biochim Biophys Acta 1242: 29-41, 1995.

11. Chintala SK, Fueyo J, Gomez-Manzano C, Venkaiah B, Bjerkvig R, Yung WK, Sawaya R, Kyritsis AP and Rao JS: Adenovirus-mediated p16/CDKN2 gene transfer suppresses glioma invasion in vitro. Oncogene 15: 2049-2057, 1997.

12. Fahraeus R and Lane DP: The p16(INK4a) tumour suppressor protein inhibits alphavbeta3 integrin-mediated cell spreading on vitronectin by blocking PKC-dependent localization of alphavbeta3 to focal contacts. EMBO J 18: 2106-2118, 1999.

13. Naruse I, Heike Y, Hama S, Mori M and Saijo N: High concentrations of recombinant adenovirus expressing p16 gene induces apoptosis in lung cancer cell lines. Anticancer Res 18: 4275-4282, 1998. 
14. Sandig V, Brand K, Herwig S, Lukas J, Bartek J and Strauss M: Adenovirally transferred p16INK4/CDKN2 and p53 genes cooperate to induce apoptotic tumor cell death. Nat Med 3: 313-319, 1997.

15. Ploug M: Structure-function relationships in the interaction between the urokinase-type plasminogen activator and its receptor. Curr Pharm Des 9: 1499-1528, 2003.

16. Rao JS: Molecular mechanisms of glioma invasiveness: the role of proteases. Nat Rev Cancer 3: 489-501, 2003.

17. Degryse B, Resnati M, Czekay RP, Loskutoff DJ and Blasi F: Domain 2 of the urokinase receptor contains an integrin-interacting epitope with intrinsic signaling activity: generation of a new integrin inhibitor. J Biol Chem 280: 24792-24803, 2005.

18. Aguirre Ghiso JA, Kovalski K and Ossowski L: Tumor dormancy induced by downregulation of urokinase receptor in human carcinoma involves integrin and MAPK signaling. J Cell Biol 147: 89-104, 1999.

19. Pollanen J, Hedman K, Nielsen LS, Dano K and Vaheri A: Ultrastructural localization of plasma membrane-associated urokinase-type plasminogen activator at focal contacts. J Cell Biol 106: 87-95, 1988.

20. Wei Y, Czekay RP, Robillard L, Kugler MC, Zhang F, Kim KK, Xiong JP, Humphries MJ and Chapman HA: Regulation of alpha5betal integrin conformation and function by urokinase receptor binding. J Cell Biol 168: 501-511, 2005.

21. Wei Y, Lukashev M, Simon DI, Bodary SC, Rosenberg S, Doyle MV and Chapman HA: Regulation of integrin function by the urokinase receptor. Science 273: 1551-1555, 1996

22. Xue W, Mizukami I, Todd RF III and Petty HR: Urokinasetype plasminogen activator receptors associate with beta1 and beta3 integrins of fibrosarcoma cells: dependence on extracellular matrix components. Cancer Res 57: 1682-1689, 1997.

23. Yebra M, Goretzki L, Pfeifer M and Mueller BM: Urokinasetype plasminogen activator binding to its receptor stimulates tumor cell migration by enhancing integrin-mediated signal transduction. Exp Cell Res 250: 231-240, 1999.

24. Bello L, Francolini M, Marthyn P, Zhang J, Carroll RS, Nikas DC, Strasser JF, Villani R, Cheresh DA and Black PM: Alpha (v)beta3 and alpha(v)beta5 integrin expression in glioma periphery. Neurosurgery 49: 380-389, 2001.

25. Adachi Y, Lakka SS, Chandrasekar N, Yanamandra N, Gondi CS, Mohanam S, Dinh DH, Olivero WC, Gujrati M, Tamiya T, Ohmoto T, Kouraklis G, Aggarwal B and Rao JS: Downregulation of integrin alpha(v)beta(3) expression and integrinmediated signaling in glioma cells by adenovirus-mediated transfer of antisense urokinase-type plasminogen activator receptor (uPAR) and sense p16 genes. J Biol Chem 276: 47171-47177, 2001.

26. Adachi Y, Chandrasekar N, Kin Y, Lakka SS, Mohanam S, Yanamandra N, Mohan PM, Fuller GN, Fang B, Fueyo J, Dinh DH, Olivero WC, Tamiya T, Ohmoto T, Kyritsis AP and Rao JS: Suppression of glioma invasion and growth by adenovirus-mediated delivery of a bicistronic construct containing antisense uPAR and sense p16 gene sequences. Oncogene 21: 87-95, 2002.
27. Milner R and Ffrench-Constant C: A developmental analysis of oligodendroglial integrins in primary cells: changes in alpha $\mathrm{v}-$ associated beta subunits during differentiation. Development 120: 3497-3506, 1994.

28. Milner R, Wilby M, Nishimura S, Boylen K, Edwards G, Fawcett J, Streuli C, Pytela R and Ffrench-Constant C: Division of labor of Schwann cell integrins during migration on peripheral nerve extracellular matrix ligands. Dev Biol 185: 215-228, 1997.

29. Green DR: Apoptotic pathways: ten minutes to dead. Cell 121: 671-674, 2005.

30. Korsmeyer SJ: BCL-2 gene family and the regulation of programmed cell death. Cancer Res 59: 1693S-1700S, 1999.

31. Tan Y, Demeter MR, Ruan H and Comb MJ: BAD Ser-155 phosphorylation regulates $\mathrm{BAD} / \mathrm{Bcl}-\mathrm{XL}$ interaction and cell survival. J Biol Chem 275: 25865-25869, 2000.

32. Yang E, Zha J, Jockel J, Boise LH, Thompson CB and Korsmeyer SJ: Bad, a heterodimeric partner for Bcl-XL and Bcl-2, displaces Bax and promotes cell death. Cell 80: 285-291, 1995.

33. Zha J, Harada H, Yang E, Jockel J and Korsmeyer SJ: Serine phosphorylation of death agonist BAD in response to survival factor results in binding to $14-3-3$ not BCL-X(L). Cell 87: 619-628, 1996.

34. Datta SR, Dudek H, Tao X, Masters S, Fu H, Gotoh Y and Greenberg ME: Akt phosphorylation of BAD couples survival signals to the cell-intrinsic death machinery. Cell 91: 231-241, 1997.

35. Del Peso L, Gonzalez-Garcia M, Page C, Herrera R and Nunez G: Interleukin-3-induced phosphorylation of BAD through the protein kinase Akt. Science 278: 687-689, 1997.

36. Kennedy SG, Kandel ES, Cross TK and Hay N: Akt/protein kinase B inhibits cell death by preventing the release of cytochrome c from mitochondria. Mol Cell Biol 19: 5800-5810, 1999.

37. Khwaja A: Akt is more than just a bad kinase. Nature 401: 33-34, 1999.

38. Sakurai S, Sonoda Y, Koguchi E, Shinoura N, Hamada H and Kasahara T: Mutated focal adhesion kinase induces apoptosis in a human glioma cell line, T98G. Biochem Biophys Res Commun 293: 174-181, 2002.

39. Sonoda Y, Watanabe S, Matsumoto Y, Izu-Yokota E and Kasahara T: FAK is the upstream signal protein of the phosphatidylinositol 3-kinase-Akt survival pathway in hydrogen peroxide-induced apoptosis of a human glioblastoma cell line. J Biol Chem 274: 10566-10570, 1999.

40. Plate KH, Breier G, Weich HA and Risau W: Vascular endothelial growth factor is a potential tumour angiogenesis factor in human gliomas in vivo. Nature 359: 845-848, 1992. 